\title{
Treatment of Unilateral PA by Adrenalectomy: Potential Reasons for Incomplete Biochemical Cure
}

\author{
Authors \\ Yuhong Yang', Martin Reincke', Tracy Ann Williams 1, 2
}

\author{
Affiliations \\ 1 Medizinische Klinik und Poliklinik IV, Klinikum der \\ Universität München, Ludwig-Maximilians-Universität \\ München, Munich, Germany \\ 2 Division of Internal Medicine and Hypertension, Depart- \\ ment of Medical Sciences, University of Turin, Turin, Italy
}

\section{Key words}

Primary aldosteronism, adrenalectomy, aldosterone-producing adenoma, bilateral adrenal hyperplasia, persistent hyperaldosteronism

$\begin{array}{ll}\text { received } & 23.05 .2018 \\ \text { revised } & 11.07 .2018 \\ \text { accepted } & 23.07 .2018\end{array}$

\section{Bibliography}

DOI https://doi.org/10.1055/a-0662-6081

Published online: 21.8 .2018

Exp Clin Endocrinol Diabetes 2019; 127: 100-108

(c) J. A. Barth Verlag in Georg Thieme Verlag KG

Stuttgart · New York

ISSN 0947-7349

\section{Correspondence}

Tracy Ann Williams

Medizinische Klinik und Poliklinik IV

Klinikum der Universität München

LMU München

Ziemssenstr. 1

80336 München

Germany

Tel.: + 49/89/4400 52941, Fax: + 49/89/4400 54428

Tracy.Williams@med.uni-muenchen.de

\begin{abstract}
The importance of an early diagnosis and appropriate management of patients with primary aldosteronism (PA) has become increasingly clear because of the adverse impact of the disorder on cardiovascular and cerebrovascular events and target organ damage. Adrenalectomy potentially cures patients with unilateral PA resulting in normalisation of blood pressure or significant clinical improvements in the majority of patients. Different criteria have been used to evaluate outcomes of unilateral adrenalectomy. Clinical remission (cure of hypertension) is observed in $6 \%$ to $86 \%$ of patients and clinical benefits from surgery are seen in the majority. Several factors have been identified that predict clinical success after surgery such as age, sex, anti-hypertensive medication dosage and known duration of hypertension. Biochemical remission of PA after unilateral adrenalectomy, characterised by the resolution of hyperaldosteronism and correction of pre-surgical hypokalaemia, is observed in $67 \%$ to $100 \%$ of patients with unilateral PA. In only a small proportion of patients, adrenalectomy fails to resolve hyperaldosteronism and inappropriate aldosterone production persists after surgery. In this review we discuss the potential reasons for failing to cure hyperaldosteronism after unilateral adrenalectomy for unilateral primary aldosteronism.
\end{abstract}

\section{Introduction}

Primary aldosteronism (PA) is a heterogeneous syndrome characterized by hypertension, aldosterone overproduction and suppressed plasma renin [1]. Spontaneous hypokalaemia was originally regarded as a critical feature, but it is now known that most patients with PA are normokalaemic (63-91\%) [2]. The prevalence of PA is estimated as $6 \%$ in the general population with hypertension [1], $11 \%$ in patients referred to hypertension centres [3], and
$15-20 \%$ in patients with resistant hypertension [4]. PA is now recognised as the most frequent form of endocrine hypertension with unilateral PA the most common surgically curable form of hypertension.

Patients with PA display an elevated risk of cardiovascular and cerebrovascular diseases, target organ damage to the heart and kidney and metabolic alterations, including metabolic syndrome and diabetes [5-9], compared with patients with primary hyper- 
tension with matched cardiovascular risk profiles [10]. These detrimental effects are caused by the excessive secretion of aldosterone and a potential role for glucocorticoid overproduction has also been reported $[8,11]$. PA is also associated with psychological symptoms such as anxiety and depression with a negative impact on quality of life $[12,13]$. Therefore, an early diagnosis and specific treatment of unilateral and bilateral PA is essential.

The diagnostic work-up of PA is recommended by the Endocrine Society's Clinical Guideline and begins with the screening for inappropriately elevated aldosterone production relative to suppressed plasma renin levels (aldosterone-to-renin ratio, ARR) in most patients with hypertension except those with mild hypertension [14]. Patients with a positive ARR test proceed to confirmation testing (including oral sodium loading, saline infusion, fludrocortisone suppression, and captopril challenge) and finally subtype differentiation of unilateral from bilateral PA (including adrenal venous sampling (AVS) and computed tomography (CT) scanning) [15]. The clinical management of PA depends on subtype differentiation: mineralocorticoid receptor (MR) antagonists for patients with bilateral PA, and unilateral adrenalectomy for patients with unilateral forms (including unilateral aldosterone producing adenomas (APA) and unilateral adrenal hyperplasia) [15].

Laparoscopic adrenalectomy is the recommended surgical treatment option due to the associated low morbidity (5-14\%), mortality well below $1 \%$ and short hospital stays of usually 3 days [16]. Surgical resection of the hyperfunctioning adrenal potentially normalises blood pressure (BP) and should reverse the detrimental effects of hormonal excess [13, 16-18]. Clinical improvements, with significant post-surgical reductions in blood pressure, are observed in most patients such that a clinical benefit (complete clinical success [clinical remission] or partial clinical success [clinical improvement]) is achievable in over 4 out of every 5 patients [18]. Reasons contributing to the absence of clinical remission include the presence of background primary hypertension, long-standing hypertension, older age, renal insufficiency or other co-morbidities [19-24]. In contrast, biochemical remission with cure of hyperaldosteronism and normalisation of spontaneous hypokalaemia (if present pre-surgically) should be achieved in all patients with a correct diagnosis of unilateral PA and surgically successful total adrenalectomy. Despite this, in a recent multicentre international co- hort study, persistent biochemical aldosteronism after adrenalectomy was observed in $6 \%$ of 699 patients with a diagnosis of unilateral PA by AVS [18]. In this manuscript, we review outcomes after adrenalectomy and possible reasons for incomplete biochemical remission after total adrenalectomy, summarised in $>$ Table 1.

\section{Surgical and Medical Management of PA}

Adrenalectomy has a low frequency of major complications (1.3\%) [25] and is associated with a high proportion of patients achieving biochemical remission $[16,18,26-28]$. Several studies report significant improvements in quality of life in patients treated surgically rather than medically [29-31]. Patients with medical management have worse quality of life scores (measured by Medical Outcomes Study Short Form 36 General Health Survey) with increased depression and anxiety compared with patients with surgical treatment [29, 30, 32]. A prospective study identified increased beneficial effects of surgical treatment compared with medical management on patient quality of life, with a health-related quality of life almost comparable with that of the general population [31]. This may be related to continued hormone excess in patients with medical treatment which may still affect mood-regulating areas of the brain, and cortisol co-secretion from an APA may impair psychological well-being [33].

A study that compared ten-year follow-up between 602 patients initiated on MR antagonists and 41,853 age-matched patients with primary hypertension demonstrated doubling the incidence of cardiovascular events in patients with PA treated with MR antagonists, despite comparable blood pressure levels [34]. The risks of incident diabetes, atrial fibrillation, and mortality were also increased in patients with MR antagonist treatment. The adverse cardiometabolic outcomes of patients with PA treated with MR antagonists was limited to patients with sustained renin activity suppression $(<1 \mu \mathrm{g} / \mathrm{L} / \mathrm{h})$, therefore the study highlighted the importance of optimal titration of MR antagonist dosage and long-term follow-up with monitoring of biochemical parameters [34]. The comparison of kidney function between surgically and medically treated patients with PA reported a decrease in GFR and albumin excretion with surgical treatment compared with medical treatment with low-dose spironolactone (50 mg/day) [35, 36]. Moreover, a large

- Table 1 Treatment of unilateral PA by adrenalectomy: potential reasons for incomplete biochemical cure.

\begin{tabular}{|c|c|}
\hline \multirow[t]{2}{*}{$\begin{array}{l}\text { Adrenalectomy of } \\
\text { non-dominant } \\
\text { (contralateral) adrenal }\end{array}$} & $\begin{array}{l}\text { Subtype differentiation by CT scanning: } \\
\text { 1) lacks sensitivity to detect micro-APAs }<10 \mathrm{~mm} \text { in diameter } \\
\text { 2) lack of functional information can result in resection of incidentaloma instead of source of aldosterone production }\end{array}$ \\
\hline & $\begin{array}{l}\text { Subtype differentiation by AVS: } \\
\text { 1) influence of protocol: } \\
\text { - sequential unstimulated AVS could result in artificial gradients between adrenal glands in prolonged procedures } \\
\text { 2) interpretation of results: } \\
\quad \text { - less strigent criteria for SI and LI can compromise diagnostic accuracy }\end{array}$ \\
\hline $\begin{array}{l}\text { Adrenalectomy of } \\
\text { dominant (ipsilateral) } \\
\text { adrenal }\end{array}$ & Bilateral PA with asymmetrical production of aldosterone can potentially give an AVS result of unilateral PA. \\
\hline \multicolumn{2}{|c|}{$\begin{array}{l}\text { APA, aldosterone-producing adenoma; ACTH, adrenocorticotropic hormone; AVS, adrenal venous sampling; CT, computed tomography; LI, } \\
\text { lateralisation index; PA, primary aldosteronism; SI, selectivity index; Potential reasons for failing to cure hyperaldosteronism after adrenalectomy for } \\
\text { unilateral PA are shown. These include adrenalectomy on the wrong side resulting from limitations of CT scanning (lack of sensitivity and functional } \\
\text { information) and AVS (influence of protocol and interpretation of results) or adrenalectomy of ipsilateral adrenal in patients with bilateral PA with } \\
\text { asymmetrical production of aldosterone. }\end{array}$} \\
\hline
\end{tabular}


prospective study that assessed long-term cardiovascular outcomes and mortality with a median of 11.8 years follow-up reported a higher potential of atrial fibrillation and lower atrial fibrillationfree survival in medically treated patients [37]. In contrast, the long-term outcomes of surgically treated patients were similar to those of patients with primary hypertension [37].

\section{Post-Operative Outcomes of Adrenalectomy for Unilateral PA}

Surgical management of unilateral PA resolves the biochemical abnormalities such as elevated aldosterone production, abnormal ARR and hypokalaemia (if present pre-surgically) in $67 \%-100 \%$ of patients $[16,18,26-28,38]$. For clinical outcomes, the proportion of patients achieving cure of elevated blood pressure is highly variable, ranging from $6 \%$ to $86 \%$, with an improvement reported in $14 \%-85 \%$ of patients $[13,16,18,26-28,38]$. A decrease in systolic $\mathrm{BP}$ of 25 to $40 \mathrm{mmHg}$ and a reduction in antihypertensive medica- tion dosage (1-2 drug classes) have been reported after adrenalectomy for unilateral PA $[38,39]$. A reduction in left ventricular hypertrophy has also been reported at short-term and long-term follow-up [40]. Glomerular hyperfiltration (an increased glomerular filtration rate (GFR) and decreased urinary albumin excretion) is reversible by surgery $[35,41,42]$ (

The above studies used diverse diagnostic procedures, different criteria and duration of follow-up to assess post-surgical outcomes. The PASO (primary aldosteronism surgical outcome) study [18] established a set of internationally standardised criteria for outcome assessment in which outcomes were defined as complete (cure or remission), partial (improvement) and absent (failure) clinical or biochemical success with outcome assessment at 6-12 months. These criteria were then used to assess outcomes in an international multicentre cohort of 705 patients with subtype diagnosis by AVS. Biochemical remission classified as normalisation of plasma potassium (if hypokalaemia was present pre-surgery) and of the ARR was achieved in $94 \%$ of patients, with the remaining patients

- Table 2 Cardiometabolic events and target organ damage in unilateral PA versus essential hypertension at diagnosis and follow-up after unilateral adrenalectomy.

\begin{tabular}{|c|c|c|c|c|c|c|}
\hline \multirow{2}{*}{$\begin{array}{l}\text { Cardiometabolic Events and Target } \\
\text { Organ Damage }\end{array}$} & \multicolumn{2}{|c|}{ Diagnosis } & \multicolumn{2}{|c|}{ FU } & \multirow{2}{*}{$\begin{array}{c}\text { FU } \\
\text { Interval }\end{array}$} & \multirow[t]{2}{*}{ Reference } \\
\hline & Unilateral PA & EH & Unilateral PA & EH & & \\
\hline \multirow[t]{4}{*}{ Stroke and/or TIA } & $24 / 224(11 \%)$ & $12 / 224(5 \%)$ & & & & [81] \\
\hline & $3 / 29(10 \%)$ & $9 / 323(3 \%)$ & & & & {$[9]$} \\
\hline & $7 / 57(12 \%)$ & $5 / 171(3 \%)$ & $3 / 57(5 \%)$ & $2 / 171(1 \%)$ & $12 \mathrm{y}$ & [5] \\
\hline & $0 / 41(0 \%)$ & $0 / 894(0 \%)$ & $1 / 34(3 \%)$ & $7 / 700(1 \%)$ & $11.8 \mathrm{y}$ & [37] \\
\hline \multirow[t]{4}{*}{ CAD (MI and/or Reversible Ischemia) } & $4 / 224(2 \%)$ & 9/224 (4\%) & & & & [81] \\
\hline & $6 / 29(21 \%)$ & $27 / 323(8 \%)$ & & & & [9] \\
\hline & $3 / 57(5 \%)$ & $4 / 171(2 \%)$ & $1 / 57(2 \%)$ & $3 / 171(2 \%)$ & $12 y$ & [5] \\
\hline & $0 / 41(0 \%)$ & $0 / 894(0 \%)$ & $1 / 34(3 \%)$ & $26 / 684(4 \%)$ & $11.8 y$ & [37] \\
\hline \multirow[t]{3}{*}{ Arrhythmias } & $5 / 29(17 \%)$ & $11 / 323(3 \%)$ & & & & [81] \\
\hline & $5 / 57(9 \%)$ & $1 / 171(0.6 \%)$ & $1 / 57(2 \%)$ & $3 / 171(2 \%)$ & $12 y$ & [5] \\
\hline & $2 / 41(5 \%)$ & $2 / 894(0.2 \%)$ & $2 / 35(6 \%)$ & $20 / 667(3 \%)$ & $11.8 \mathrm{y}$ & [37] \\
\hline \multirow[t]{3}{*}{ Heart Failure } & $8 / 224(4 \%)$ & 9/224 (4\%) & & & & [81] \\
\hline & $1 / 57(2 \%)$ & $1 / 171(0.6 \%)$ & $2 / 57(4 \%)$ & $0 / 171(0 \%)$ & $12 \mathrm{y}$ & {$[5]$} \\
\hline & $0 / 41(0 \%)$ & $0 / 894(0 \%)$ & $2 / 35(6 \%)$ & $10 / 667(2 \%)$ & $11.8 \mathrm{y}$ & [37] \\
\hline \multirow[t]{3}{*}{ LVH } & $9 / 24(38 \%)$ & $24 / 108(22 \%)$ & $2 / 24(8 \%)$ & 9/108 (8\%) & $6.4 y$ & {$[82]$} \\
\hline & $67 / 110(61 \%)$ & 76/143 (53\%) & $50 / 110(46 \%)$ & $61 / 143(43 \%)$ & $3 y$ & {$[40]$} \\
\hline & $17 / 26(65 \%)$ & $390 / 1229(32 \%)$ & & & & {$[1]$} \\
\hline PAD & $2 / 29(7 \%)$ & $8 / 323(2 \%)$ & & & & [9] \\
\hline \multirow[t]{3}{*}{ Metabolic Syndrome } & $11 / 37(30 \%)$ & $39 / 192(20 \%)$ & & & & [83] \\
\hline & $6 / 9(67 \%)$ & $4 / 11(36 \%)$ & $5 / 9(56 \%)$ & NA & $6 \mathrm{~m}$ & {$[84]$} \\
\hline & $12 / 27(44 \%)$ & $441 / 1478(30 \%)$ & & & & [1] \\
\hline \multirow[t]{2}{*}{ Diabetes } & $11 / 50(22 \%)$ & $20 / 90(22 \%)$ & & & & {$[85]$} \\
\hline & $0 / 27(0 \%)$ & $55 / 1414(4 \%)$ & & & & {$[1]$} \\
\hline Microalbuminuria & $6 / 21(29 \%)$ & $150 / 1168(13 \%)$ & & & & {$[1]$} \\
\hline \multicolumn{7}{|c|}{$\begin{array}{l}\text { CAD, coronary artery disease; EH, essential hypertension; FU, follow-up; LVH, left ventricular hypertrophy; m, months; MI, myocardial infarction; NA, } \\
\text { not available; PA, primary aldosteronism; PAD, peripheral arterial disease; TIA, transient ischaemic attack; y, years. Values indicate numbers of } \\
\text { patients with cardiometabolic events or target organ damage divided by total number of patients with the proportion of affected patients in } \\
\text { parenthesis; The incidence of cardiometabolic events and target organ damage are shown for patients with unilateral PA compared with patients with } \\
\text { essential hypertension at diagnosis and follow-up after adrenalectomy. The cardiometabolic events and target organ damage include stroke and/or } \\
\text { transient ischaemic attack, coronary artery disease, arrhythmias, heat failure, left ventricular hypertrophy, peripheral arterial disease, metabolic } \\
\text { syndrome, diabetes, and microalbuminuria. }\end{array}$} \\
\hline
\end{tabular}


comprising those with partial biochemical success (4\%) and those with absent success ( $2 \%)$. In contrast, the proportion of patients with complete clinical success (cure of hypertension) was $37 \%$, with $47 \%$ of patients attaining significant clinical improvements. Female sex and younger age were associated with clinical benefit (complete + partial clinical success) with sex and age contributing to the diverse between-centre clinical outcomes. Miller et al., evaluated the PASO standards against other outcome criteria and reported an improvement in the definition of short- and long-term post-surgical outcomes with the PASO consensus [28].

\section{Post-Surgical Persistent Hyperaldosteronism: Differentiation of Unilateral from Bilateral PA by Adrenal CT Scanning}

\section{Subtype differentiation by adrenal CT scanning}

The successful treatment by adrenalectomy is dependent on the accurate differentiation of unilateral from bilateral PA. The most widely diffuse approach for subtype determination is CT or magnetic resonance imaging (MRI) of the adrenals to detect morphological alterations followed by AVS in centres where it is available. The Endocrine Society's Clinical Guideline recommends adrenal CT on all patients with confirmed PA [14] to exclude adrenal carcinomas that are usually $>40 \mathrm{~mm}$ in diameter and differ in densitometry from adenomas $[43,44]$. CT scanning also helps delineate the anatomy of adrenal veins to aid positioning of catheters in AVS. However, the sensitivity of CT is often inadequate for detection of micro-APAs $<10 \mathrm{~mm}$ in diameter, and an APA can display like small hypodense (normally lower than $10 \mathrm{HU}$ ) nodules on unenhanced CT due to its high component of intracellular lipid [14,44]. Therefore, small APAs can be undetectable on CT, or can be misinterpreted by radiologists as idiopathic hyperaldosteronism when coincidently detecting bilateral nodularity or normal-appearing adrenals [14]. Moreover, CT cannot provide functional information on aldosterone secretion. Non-functioning adenomas (or incidentalomas) share a similar morphology to APA on CT and are difficult to differentiate, which is a problem compounded in older individuals $>35$ years old who more frequently have incidentalomas [45]. Thus, inappropriate adrenalectomy of a functionally normal adrenal can occur if solely relying on CT scanning.

\section{Performance of adrenal CT scanning compared with AVS}

A number of studies have compared adrenal imaging with AVS for the accuracy of lateralisation with some studies assessing the histology of the resected adrenal. Gordon RD et al., [46] reported correct $C T$ lateralisation in around half $(59 / 111)$ of patients and $<25 \%$ in patients with an APA $<10 \mathrm{~mm}$ in diameter, and a report from the Mayo Clinic showed that unnecessary adrenalectomies could occur in $25 \%$ of patients whilst $22 \%$ of patients could miss the chance of surgery [17]. In a systematic review, the proportion of patients with a misdiagnosis of PA subtypes was approximately $40 \%$ when relying on CT and MRI alone by comparison with AVS results as the reference standard [47]. A study comparing imaging and AVS results in 263 patients revealed that adrenal imaging and AVS were concordant to the surgically documented side in $58.6 \%$ and $97.1 \%$ of patients, respectively [27]. Another study included 175 patients who underwent unilateral adrenalectomy reported the accuracy of CT as $57.3 \%$ for detecting unilateral PA, with $12.2 \%$ of contralateral (non-dominant) side of lateralisation and $22 \%$ of false diagnosis of bilateral PA, while AVS results could prove unilateral aldosterone hypersecretion correctly [48]. A recent study compared AVS with CT data in patients with unilateral PA (diagnosed by AVS) and biochemical cure at follow-up and indicated that imaging and AVS results were concordant in $64 \%$ of 491 patients with $34 \%$ of patients showing bilateral normal or bilateral abnormal results by CT scanning and $2 \%$ of patients with unilateral abnormal imaging of the contralateral adrenal [49]. All these studies therefore indicated the superior performance of AVS to CT and MRI techniques for subtype differentiation of PA. However, a restricted subgroup of patients can bypass AVS using predictors of younger age and marked phenotype of PA [14]. A high accuracy (100\%) of CT scanning has been demonstrated for lateralisation in PA with a large nodule $>10 \mathrm{~mm}$ in diameter with normal appearance of the contralateral gland in young patients (aged $<35$ years) with spontaneous hypokalaemia and marked aldosterone excess at baseline $[49,50]$. A high accuracy ( $87 \%$ ) was also noted in patients aged 35-40 years with evident symptoms [50]. Therefore, with the exception of young patients with a specific phenotype, most retrospective studies indicate that CT imaging is not as reliable as AVS for accurate subtype differentiation in PA.

A recent randomized prospective trial compared the treatment outcomes in patients with CT- and AVS-based management and challenged the reference standard status of AVS [51]. A total of 184 patients with florid PA were randomly assigned to CT-based and AVS-based treatments and outcomes were assessed at one year of follow-up. The study reported no differences in antihypertensive medication dosage (median 3.0 vs 3.0), normalisation of target blood pressure ( $42 \%$ vs $45 \%$ ), and medians of RAND-36 physical or mental score ( 52.7 vs 53.2) between two groups and concluded that CT or AVS management did not result in significant differences in clinical benefits after one-year follow-up. Biochemical remission was $80 \%$ with CT-based management (vs $89 \%$ in AVS-based management) [51]. Similar conclusions were also made by a retrospective study that reported comparable follow-up blood pressures in 109 patients with CT-based and 59 patients with AVS-based adrenalectomy [52]. The prospective clinical trial was criticised for being underpowered with a non-representative study cohort due to inclusion of fewer females and florid PA that can predict persistence of high blood pressure due to target organ damage. The use of cosyntropin in the AVS procedure was also criticised because this can confound or even invert lateralisation thereby indicating adrenalectomy on the wrong side [53]. A retrospective assessment of the post-surgical outcomes of 761 patients treated by unilateral adrenalectomy for unilateral PA (235 and 526 patients with a surgical approach based on CT or AVS, respectively) in accordance with the PASO criteria reported that CT-based surgical management was a predictor of a decreased likelihood of complete biochemical success, and was associated with inappropriate post-surgical aldosterone production and absent clinical success [49]. 
Post-Surgical Persistent Hyperaldosteronism: Differentiation of Unilateral from Bilateral PA by Adrenal Venous Sampling

\section{Influence of AVS protocol: unstimulated versus ACTH stimulated AVS}

Adrenocorticotropic hormone (ACTH) levels fluctuate in a diurnal pattern and increase when the hypothalamic-pituitary-adrenal axis is activated by emotional and pain-related stress thereby affecting both aldosterone and cortisol secretion. AVS can employ ACTH (124) (cosyntropin, a synthetic derivative of ACTH that contains the first 24 amino acids but maintains full function) stimulation by either constant cosyntropin infusion $(50 \mu \mathrm{g} / \mathrm{h}$, started $30 \mathrm{~min}$ before AVS) or by bolus (usually $0.25 \mathrm{mg}$ ) [54].

ACTH-stimulated AVS enhances the selectivity index (SI, ratio of concentrations of cortisol from an adrenal vein and a peripheral vein) by maximising the cortisol gradients between adrenal and peripheral veins, thereby increasing success rates of sampling which especially benefits less experienced radiologists [55]. Because ACTH minimizes cortisol fluctuations, AVS with sequential catheterisation can be performed [55]. Furthermore, additional cortisol secretion provides steroid prophylaxis for patients with high risks of allergic reaction. By contrast, unstimulated AVS requires additional patient management. Without cosyntropin stimulation, patients are suggested to be sustained in the supine position for one hour before AVS which should be performed in the early morning when the ACTH secretion is highest to avoid false-negative results [56]. Besides, measures solving emotion- and pain-related stress should be adopted such as reassurance from doctors, administration of benzodiazepines and local anesthesia [55, 57]. Unstimulated AVS should also require bilateral simultaneous catheterisation [58].

Studies that compared unstimulated with ACTH-stimulated AVS highlighted the potential pitfalls introduced by ACTH stimulation. It not only increases aldosterone production from APAs, but will also stimulate aldosterone secretion from the contralateral adrenal, thereby potentially reducing the lateralisation index ( $\mathrm{LI}$, aldosterone concentrations divided by the respective cortisol concentrations between adrenal veins) and introducing the possibility of a false diagnosis of bilateral PA [58]. A multicentre study that compared basal and ACTH stimulated AVS (by ACTH infusion or bolus) reported a significant increase in SI especially after ACTH bolus but no differences in $\mathrm{LI}$ between the two approaches. The discordant diagnoses between basal and post-ACTH conditions ranged from $17 \%$ to $36 \%$ depending on the criteria, with the more stringent criteria contributing to the higher reproducibility of diagnosis [59]. Another study with 171 patients with AVS performed before and after ACTH bolus found a discordance in subtype diagnosis of $28 \%$, most of which were lateralized cases under basal conditions that were diagnosed as bilateral after ACTH stimulation [60]. A higher rate $(91 \%)$ of concordant diagnoses was obtained by Wolley et al. [61], in which one unilateral case was diagnosed as bilateral and two bilateral cases became unilateral after ACTH stimulation.

Hence, ACTH-stimulated AVS is useful to reduce non-diagnostic cases, allows sequential catheterisation, and benefits patients with an allergic reaction or when AVS cannot be performed in the morning. However, there is a potential to confound lateralisation results and therefore the protocol requires the optimization of diagnostic criteria.

\section{Influence of AVS protocol: simultaneous unstimulated versus sequential unstimulated AVS}

Oscillations of aldosterone and cortisol secretion can produce artificial gradients between adrenal glands if AVS is performed sequentially under basal conditions. This is circumvented by simultaneous cannulation but the possibility of adrenal vein thrombosis is increased because the procedure requires obstruction of vessel lumen by the catheter until achieving successful cannulation of the contralateral vein. Consequently simultaneous bilateral AVS is more invasive and technically demanding and there is an inverse correlation of the rate of complications and the number of AVS performed by radiologists [62].

A study investigating the effect of stress on SI alterations during sequential and simultaneous AVS demonstrated that the SI from sequential AVS is higher than with simultaneous AVS, indicating differences of selectivity between the two methods of cannulation [63]. However, in the PASO study, the Brisbane group used a sequential unstimulated AVS approach with no absent biochemical success cases, which argues against important fluctuations of lateralisation of gradients due to stress or pulsatile secretion [18]. Moreover, a retrospective study compared simultaneous and sequential unstimulated AVS in 188 patients. Bilateral simultaneous AVS were obtained twice within five minutes, whilst sequential AVS was performed with a time-gap of 5 min between the two catheterisations. No significant difference was recorded in terms of the $\mathrm{SI}$ and $\mathrm{LI}$ values between simultaneous and sequential AVS and between two repeated simultaneous AVS. It suggested no superiority of simultaneous over sequential AVS under basal conditions if catheterisation of two adrenal veins can be achieved within five minutes [64].

Therefore, there is no consensus on the better performance of simultaneous or sequential unstimulated AVS, but the two approaches have a similar accuracy when performed by skilled and experienced radiologists if the sequential approach is performed in a short time interval. However, simultaneous unstimulated AVS is more likely to be preferred, considering potential fluctuations of aldosterone and cortisol during sequential catheterisation in an unstimulated AVS procedure.

\section{Interpretation of AVS results}

AVS results are interpreted by calculating several indexes based on aldosterone and cortisol concentrations in the adrenal or peripheral veins, and the cut-offs employed define the diagnosis.

$\mathrm{SI}$ is used to confirm adequate cannulation of adrenal veins on the assumption that cortisol is exclusively produced from adrenals and is not overproduced by an APA. No consensus has been reached on the criteria for successful catheterisation. The cut-offs of the SI vary among referral centres, ranging from 1.1 to 3 under basal conditions and 2 to 5 with ACTH stimulated AVS [65]. Expert recommendations for $S I$ cut-offs have been proposed as $\geq 2$ for unstimulated AVS, and $\geq 3.0$ for AVS with ACTH stimulation [55], and $>3$ under basal conditions and $>5$ in ACTH stimulated AVS [54]. Most centres adopt $\mathrm{SI}>2$ to 3 in basal conditions and $>3$ to 5 with ACTH stimulation [66], and considerable disparities in diagnosis are 
reached using less stringent criteria for successful cannulation. A study compared the influences of permissive $(S I \geq 1.1)$, intermediate ( $S I \geq 2)$ and strict $(S I \geq 3)$ criteria on subtype diagnosis in unstimulated AVS. Permissive criteria achieved the highest success rate of bilateral cannulation ( $91 \%$ ), while the proportion of concordant diagnoses was decreased to $35 \%$ between the first and second AVS. It was proposed that an SI cut-off of $\geq 2.75$ is necessary for diagnostic reproducibility [67]. These three criteria were compared again under basal AVS. It showed that the permissive cut-off values were associated with the lowest specificity (76.9\%) and highest sensitivity (98.5\%) for selectivity, whilst the strict criteria guaranteed $100 \%$ specificity but compromised sensitivity (50.4\%). A SI cut-off of 2 was finally recommended by this study to gain best sensitivity (70.8\%) and $100 \%$ specificity [68]. To identity the most accurate criteria for AVS, a study analysed 10 reported AVS criteria from the literature in a cohort of 108 patients with PA. An SI $>5$ was the most accurate with $88 \%$ true positives and an $\mathrm{SI}>1.1$ was accurate without ACTH stimulation [65]. Thus, a lower SI chosen for selective cannulation can increase the proportion of patients referred to lateralisation, but potentially compromise diagnostic accuracy with an impact on biochemical outcomes after adrenalectomy.

The assessment of lateralization of aldosterone hypersecretion depends on the LI. Inclusion of cortisol concentrations from adrenal veins aims to correct for the level of adrenal aldosterone in case inevitable dilution by non-adrenal blood affects their concentrations. The cut-off values for the $\mathrm{LI}$ used in referral centres vary widely. Most units use an $\mathrm{LI} \geq 4$ for the diagnosis of unilateral PA, though some centres accept more permissive criteria ( $\mathrm{LI} \geq 3$ or $\mathrm{LI} \geq 2$ ). More restrictive $\mathrm{LI}$ thresholds lead to lower rates of diagnosis of unilateral PA [67], but is more likely to select a population to be cured with adrenalectomy [55]. An LI cut-offs of 4 and 2 has been recommended for ACTH-stimulated AVS and unstimulated AVS respectively [55]. An LI $\geq 4$ with ACTH stimulation was recommended by another report which considered $\mathrm{LI}<3$ to be diagnostic of $\mathrm{BAH}$ with 3-4 a grey zone [54]. The importance of using stringent $L I$ was later highlighted by a study investigating LI from patients with hypertension with PA excluded. Lateralization gradients between 2 and 4 were found in $20 \%$ of patients with negative confirmatory test for PA, with LIs ranging from 1.01 to 3.87 [69].

Additional indexes are used in some units to replace or strengthen $\mathrm{LI}$ results for lateralisation. The utility of the ipsilateral ratio (ILR), the ratio between the aldosterone/cortisol level on the dominant side and that level in peripheral vein, together with contralateral ratio (CLR), the ratio between the aldosterone/cortisol level in contralateral adrenal vein and that level in peripheral vein, were investigated. ILR $>2$ and $C L R<1$ were regarded by some centres as a useful criterion for unilateral PA [70]. Other studies displayed a lack of effect of contralateral aldosterone suppression on outcomes of surgically treated patients with PA [71, 72]. Absolute contralateral aldosterone ratio that is aldosterone concentration in the contralateral adrenal vein divided by that in peripheral vein were set with a cut-off of 1.5 to predict outcomes after adrenalectomy [60]. Another use of aldosterone concentration is the absolute aldosterone concentration that indicates the hyperfunctioning side with aldosterone $>38780 \mathrm{pmol} / \mathrm{L}$ [73]. All these indexes are less utilized, but they can influence the cure rate after adrenalectomy in centres using and interpreting them.

\section{Can AVS fail if it has been appropriately performed and interpreted?}

AVS is however not perfect. Bilateral PA with asymmetrical production of aldosterone will potentially present an AVS result of unilateral PA when the $\mathrm{LI}$ exceeds the stringent cut-off of 4 . AVS can also give misleading results when patients with PA have concurrent subclinical Cushing's syndrome because the SI would indicate unsuccessful cannulation [74]. Patients with a successful AVS with a stringent LI (ranging from 4.4 to 10.0) still result in absent biochemical outcomes in some cases [18]. Thus, AVS cannot guarantee success even if it has been appropriately performed and interpreted.

\section{Conclusions and Perspectives}

Some patients with unilateral PA are not cured of persistent hyperaldosteronism after unilateral adrenalectomy. Focus should therefore be placed on minimizing such failures, which can be derived from inaccurate differentiation of unilateral from bilateral PA by either CT scanning or AVS. Future approaches to lateralise aldosterone production may replace or be used in conjunction with traditional methods and may minimise post-surgical biochemical persistence. Functional molecular imaging approaches using the CXCR4 ligand 68Ga-pentixafor may visualise the hypersecreting adrenal [75] as well as other functional imaging methods such as ${ }^{11} \mathrm{C}$-metomidate positron emission tomography-CT imaging and (6ß-131I) iodomethyl-19-norcholesterol scan [14]. Other approaches include potentially reducing the numbers of patients that undergo AVS by selecting patients with an APA by mass spectrometry-based peripheral venous steroid profiling $[76,77]$ or using macrolide antibiotics that selectively inhibit aldosterone hypersecretion from APAs carrying KCNJ5 mutations [78-80].[40, 81-85]

\section{Acknowledgements}

This work was supported by the European Research Council (ERC) under the European Union's Horizon 2020 research and innovation programme (grant agreement No [694913] to MR) and by the Deutsche Forschungsgemeinschaft (DFG) (within the CRC/Transregio 205/1 “The Adrenal: Central Relay in Health and Disease” to MR and TAW and grant RE 752/20-1 to MR) and the Else Kröner-Fresenius Stiftung in support of the German Conns Registry-Else-Kröner Hyperaldosteronism Registry (2013_A182 and 2015_A171 to MR). YY is supported by a fellowship from the China Scholarship Council.

\section{Conflict of Interest}

No conflict of interest has been declared by the authors.

\section{References}

[1] Monticone S, Burrello J, Tizzani D et al. Prevalence and clinical manifestations of primary aldosteronism encountered in primary care practice. J Am Coll Cardiol 2017; 69: 1811-1820

[2] Mulatero P, Stowasser M, Loh KC et al. Increased diagnosis of primary aldosteronism, including surgically correctable forms, in centers from five continents. J Clin Endocrinol Metab 2004; 89: 1045-1050 
[3] Rossi GP, Bernini G, Caliumi C et al. A prospective study of the prevalence of primary aldosteronism in 1,125 hypertensive patients. J Am Coll Cardiol 2006; 48: 2293-2300

[4] Douma S, Petidis K, Doumas M et al. Prevalence of primary hyperaldosteronism in resistant hypertension: a retrospective observational study. Lancet 2008; 371: 1921-1926

[5] Mulatero P, Monticone S, Bertello C et al. Long-term cardio- and cerebrovascular events in patients with primary aldosteronism. J Clin Endocrinol Metab 2013; 98: 4826-4833

[6] Savard S, Amar L, Plouin PF et al. Cardiovascular complications associated with primary aldosteronism: A controlled cross-sectional study. Hypertension 2013; 62: 331-336

[7] Fallo F, Veglio F, Bertello C et al. Prevalence and characteristics of the metabolic syndrome in primary aldosteronism. J Clin Endocrinol Metab 2006; 91: 454-459

[8] Arlt W, Lang K, Sitch AJ et al. Steroid metabolome analysis reveals prevalent glucocorticoid excess in primary aldosteronism. JCI Insight 2017; 2: pii: 93136

[9] Catena C, Colussi G, Nadalini E et al. Cardiovascular outcomes in patients with primary aldosteronism after treatment. Arch Intern Med 2008; 168: 80-85

[10] Monticone S, D'Ascenzo F, Moretti C et al. Cardiovascular events and target organ damage in primary aldosteronism compared with essential hypertension: A systematic review and meta-analysis. Lancet Diabetes Endocrinol 2018; 6: 41-50

[11] Funder JW. Aldosterone, sodium, and hypertension: Lessons from torcetrapib? Hypertension 2010; 55: 221-223

[12] Sonino N, Fallo F, Fava GA. Psychological aspects of primary aldosteronism. Psychother Psychosom 2006; 75: 327-330

[13] Sukor N, Kogovsek C, Gordon RD et al. Improved quality of life, blood pressure, and biochemical status following laparoscopic adrenalectomy for unilateral primary aldosteronism. J Clin Endocrinol Metab 2010; 95: 1360-1364

[14] Funder JW, Carey RM, Mantero F et al. The management of primary aldosteronism: case detection, diagnosis, and treatment: An endocrine society clinical practice guideline. J Clin Endocrinol Metab 2016; 101: 1889-1916

[15] Williams TA, Reincke M. MANAGEMENT OF ENDOCRINE DISEASE: Diagnosis and Management of Primary Aldosteronism: The Endocrine Society guideline 2016 revisited. Eur J Endocrinol 2018 pii: EJE-17-0990

[16] Muth A, Ragnarsson O, Johannsson G et al. Systematic review of surgery and outcomes in patients with primary aldosteronism. $\mathrm{Br}$ J Surg 2015; 102: 307-317

[17] Young WF, Stanson AW, Thompson GB et al. Role for adrenal venous sampling in primary aldosteronism. Surgery 2004; 136: 1227-1235

[18] Williams TA, Lenders JWM, Mulatero P et al. Outcomes after adrenalectomy for unilateral primary aldosteronism: An international consensus on outcome measures and analysis of remission rates in an international cohort. Lancet Diabetes Endocrinol 2017; 5: 689-699

[19] Kanarek-Kucner J, Stefanski A, Barraclough R et al. Insufficiency of the zona glomerulosa of the adrenal cortex and progressive kidney insufficiency following unilateral adrenalectomy - case report and discussion. Blood Press 2018; 1-9

[20] Wang W, Hu W, Zhang X et al. Predictors of successful outcome after adrenalectomy for primary aldosteronism. Int Surg 2012; 97: 104-111

[21] Waldmann J, Maurer L, Holler ] et al. Outcome of surgery for primary hyperaldosteronism. World J Surg 2011; 35: 2422-2427

[22] Lumachi F, Ermani M, Basso SM et al. Long-term results of adrenalectomy in patients with aldosterone-producing adenomas: Multivariate analysis of factors affecting unresolved hypertension and review of the literature. Am Surg 2005; 71: 864-869
[23] Meyer A, Brabant G, Behrend M. Long-term follow-up after adrenalectomy for primary aldosteronism. World J Surg 2005; 29: 155-159

[24] Proye CA, Mulliez EA, Carnaille BM et al. Essential hypertension: First reason for persistent hypertension after unilateral adrenalectomy for primary aldosteronism? Surgery 1998; 124: 1128-1133

[25] Walz MK, Alesina PF, Wenger FA et al. Posterior retroperitoneoscopic adrenalectomy - results of 560 procedures in 520 patients. Surgery 2006; 140: 943-948 discussion 948-950

[26] Citton M, Viel G, Rossi GP et al. Outcome of surgical treatment of primary aldosteronism. Langenbecks Arch Surg 2015; 400: 325-331

[27] Lim V, Guo Q, Grant CS et al. Accuracy of adrenal imaging and adrenal venous sampling in predicting surgical cure of primary aldosteronism. J Clin Endocrinol Metab 2014; 99: 2712-2719

[28] Miller BS, Turcu AF, Nanba AT et al. Refining the definitions of biochemical and clinical cure for primary aldosteronism using the primary aldosteronism surgical outcome (PASO) elstem. World J Surg 2018; 42: 453-463

[29] Kunzel HE, Apostolopoulou K, Pallauf A et al. Quality of life in patients with primary aldosteronism: Gender differences in untreated and long-term treated patients and associations with treatment and aldosterone. J Psychiatr Res 2012; 46: 1650-1654

[30] Ahmed AH, Gordon RD, Sukor $N$ et al. Quality of life in patients with bilateral primary aldosteronism before and during treatment with spironolactone and/or amiloride, including a comparison with our previously published results in those with unilateral disease treated surgically. J Clin Endocrinol Metab 2011; 96: 2904-2911

[31] Velema M, Dekkers T, Hermus A et al. Quality of life in primary aldosteronism: A comparative effectiveness study of adrenalectomy and medical treatment. J Clin Endocrinol Metab 2018; 103: 16-24

[32] Apostolopoulou K, Kunzel HE, Gerum S et al. Gender differences in anxiety and depressive symptoms in patients with primary hyperaldosteronism: a cross-sectional study. World J Biol Psychiatry 2014; 15: 26-35

[33] Reincke M. Anxiety, depression, and impaired quality of life in primary aldosteronism: Why we shouldn't ignore it!. J Clin Endocrinol Metab 2018; 103: 1-4

[34] Hundemer GL, Curhan GC, Yozamp N et al. Cardiometabolic outcomes and mortality in medically treated primary aldosteronism: A retrospective cohort study. Lancet Diabetes Endocrinol 2018; 6: 51-59

[35] Wu VC, Kuo CC, Wang SM et al. Primary aldosteronism: Changes in cystatin C-based kidney filtration, proteinuria, and renal duplex indices with treatment. J Hypertens 2011; 29: 1778-1786

[36] Wu VC, Yang SY, Lin JW et al. Kidney impairment in primary aldosteronism. Clin Chim Acta 2011; 412: 1319-1325

[37] Rossi GP, Maiolino G, Flego A et al. Adrenalectomy lowers incident atrial fibrillation in primary aldosteronism patients at long term. Hypertension 2018; 71: 585-591

[38] Steichen O, Zinzindohoue F, Plouin PF et al. Outcomes of adrenalectomy in patients with unilateral primary aldosteronism: A review. Horm Metab Res 2012; 44: 221-227

[39] Wachtel H, Cerullo I, Bartlett EK et al. Long-term blood pressure control in patients undergoing adrenalectomy for primary hyperaldosteronism. Surgery 2014; 156: 1394-1402 discussion1402-1393

[40] Rossi GP, Cesari M, Cuspidi C et al. Long-term control of arterial hypertension and regression of left ventricular hypertrophy with treatment of primary aldosteronism. Hypertension 2013; 62: 62-69

[41] Sechi LA, Di Fabio A, Bazzocchi M et al. Intrarenal hemodynamics in primary aldosteronism before and after treatment. J Clin Endocrinol Metab 2009; 94: 1191-1197

[42] Reincke M, Rump LC, Quinkler M et al. Risk factors associated with a low glomerular filtration rate in primary aldosteronism. J Clin Endocrinol Metab 2009; 94: 869-875 
[43] Young WF Jr. Conventional imaging in adrenocortical carcinoma: Update and perspectives. Horm Cancer 2011; 2: 341-347

[44] Blake MA, Cronin CG, Boland GW. Adrenal imaging. AJR Am J Roentgenol 2010; 194: 1450-1460

[45] Fassnacht M, Arlt W, Bancos I et al. Management of adrenal incidentalomas: European Society of Endocrinology Clinical Practice Guideline in collaboration with the European Network for the Study of Adrenal Tumors. Eur J Endocrinol 2016; 175: G1-G34

[46] Gordon RD, Stowasser M, Rutherford JC. Primary aldosteronism: Are we diagnosing and operating on too few patients? World J Surg 2001; 25: 941-947

[47] Kempers M], Lenders JW, van Outheusden L et al. Systematic review: Diagnostic procedures to differentiate unilateral from bilateral adrenal abnormality in primary aldosteronism. Ann Intern Med 2009; 151: 329-337

[48] Ladurner R, Sommerey S, Buechner S et al. Accuracy of adrenal imaging and adrenal venous sampling in diagnosing unilateral primary aldosteronism. Eur J Clin Invest 2017; 47: 372-377

[49] Williams TA, Burrello J, Sechi LA et al. Computed Tomography and Adrenal Venous Sampling in the Diagnosis of Unilateral Primary Aldosteronism. Hypertension 2018 pii: HYPERTENSIONAHA.118.11382

[50] Umakoshi H, Ogasawara T, Takeda Y et al. Accuracy of adrenal computed tomography in predicting the unilateral subtype in young patients with hypokalaemia and elevation of aldosterone in primary aldosteronism. Clin Endocrinol (Oxf) 2018; 88: 645-651

[51] Dekkers T, Prejbisz A, Kool LJS et al. Adrenal vein sampling versus CT scan to determine treatment in primary aldosteronism: An outcomebased randomised diagnostic trial. Lancet Diabetes Endocrinol 2016; 4: 739-746

[52] Letavernier E, Peyrard S, Amar L et al. Blood pressure outcome of adrenalectomy in patients with primary hyperaldosteronism with or without unilateral adenoma. J Hypertens 2008; 26: 1816-1823

[53] Rossi GP, Funder JW. Adrenal venous sampling versus computed tomographic scan to determine treatment in primary aldosteronism (The SPARTACUS Trial): A critique. Hypertension 2017; 69: 396-397

[54] Monticone S, Viola A, Rossato D et al. Adrenal vein sampling in primary aldosteronism: Towards a standardised protocol. Lancet Diabetes Endocrinol 2015; 3: 296-303

[55] Rossi GP, Auchus RJ, Brown M et al. An expert consensus statement on use of adrenal vein sampling for the subtyping of primary aldosteronism. Hypertension 2014; 63: 151-160

[56] Rossi GP. New concepts in adrenal vein sampling for aldosterone in the diagnosis of primary aldosteronism. Curr Hypertens Rep 2007; 9: 90-97

[57] Stowasser M, Gordon RD. Primary Aldosteronism: Changing definitions and new concepts of physiology and pathophysiology both inside and outside the kidney. Physiol Rev 2016; 96: 1327-1384

[58] Rossi GP, Pitter G, Bernante P et al. Adrenal vein sampling for primary aldosteronism: The assessment of selectivity and lateralization of aldosterone excess baseline and after adrenocorticotropic hormone (ACTH) stimulation. J Hypertens 2008; 26: 989-997

[59] Monticone S, Satoh F, Giacchetti G et al. Effect of adrenocorticotropic hormone stimulation during adrenal vein sampling in primary aldosteronism. Hypertension 2012; 59: 840-846

[60] El Ghorayeb N, Mazzuco TL, Bourdeau I et al. Basal and Post-ACTH aldosterone and its ratios are useful during adrenal vein sampling in primary aldosteronism. J Clin Endocrinol Metab 2016; 101 : 1826-1835

[61] Wolley M], Ahmed AH, Gordon RD et al. Does ACTH improve the diagnostic performance of adrenal vein sampling for subtyping primary aldosteronism? Clin Endocrinol (Oxf) 2016; 85: 703-709
[62] Rossi GP, Barisa M, Allolio B et al. The adrenal vein sampling international study (AVIS) for identifying the major subtypes of primary aldosteronism. J Clin Endocrinol Metab 2012; 97: 1606-1614

[63] Seccia TM, Miotto D, Battistel M et al. A stress reaction affects assessment of selectivity of adrenal venous sampling and of lateralization of aldosterone excess in primary aldosteronism. Eur J Endocrinol 2012; 166: 869-875

[64] Almarzooqi MK, Chagnon M, Soulez G et al. Adrenal vein sampling in primary aldosteronism: Concordance of simultaneous vs sequential sampling. Eur J Endocrinol 2017; 176: 159-167

[65] Webb R, Mathur A, Chang R et al. What is the best criterion for the interpretation of adrenal vein sample results in patients with primary hyperaldosteronism? Ann Surg Oncol 2012; 19: 1881-1886

[66] Buffolo F, Monticone S, Williams TA et al. Subtype diagnosis of primary aldosteronism: Is adrenal vein sampling always necessary? Int J Mol Sci 2017; 18: 848

[67] Mulatero P, Bertello C, Sukor N et al. Impact of different diagnostic criteria during adrenal vein sampling on reproducibility of subtype diagnosis in patients with primary aldosteronism. Hypertension 2010; 55: 667-673

[68] Mailhot JP, Traistaru M, Soulez G et al. Adrenal vein sampling in primary aldosteronism: Sensitivity and specificity of basal adrenal vein to peripheral vein cortisol and aldosterone ratios to confirm catheterization of the adrenal vein. Radiology 2015; 277: 887-894

[69] Umakoshi H, Naruse M, Wada $\mathrm{N}$ et al. Adrenal venous sampling in patients with positive screening but negative confirmatory testing for primary aldosteronism. Hypertension 2016; 67: 1014-1019

[70] Stowasser M, Gordon RD, Gunasekera TG et al. High rate of detection of primary aldosteronism, including surgically treatable forms, after 'non-selective' screening of hypertensive patients. J Hypertens 2003; 21: 2149-2157

[71] Monticone S, Satoh F, Viola A et al. Aldosterone suppression on contralateral adrenal during adrenal vein sampling does not predict blood pressure response after adrenalectomy. J Clin Endocrinol Metab 2014; 99: 4158-4166

[72] Umakoshi H, Tanase-Nakao K, Wada N et al. Importance of contralateral aldosterone suppression during adrenal vein sampling in the subtype evaluation of primary aldosteronism. Clin Endocrinol (Oxf) 2015; 83: 462-467

[73] Nishikawa T, Omura M, Satoh F et al. Guidelines for the diagnosis and treatment of primary aldosteronism--the Japan Endocrine Society 2009. Endocr J 2011; 58: 711-721

[74] Burrello J, Monticone S, Tetti M et al. Subtype Diagnosis of Primary Aldosteronism: Approach to Different Clinical Scenarios. Horm Metab Res 2015; 47: 959-966

[75] Heinze B, Fuss CT, Mulatero P et al. Targeting CXCR4 (CXC Chemokine Receptor Type 4) for Molecular Imaging of Aldosterone-Producing Adenoma. Hypertension 2018; 71: 317-325

[76] Eisenhofer G, Dekkers T, Peitzsch M et al. Mass Spectrometry-Based Adrenal and Peripheral Venous Steroid Profiling for Subtyping Primary Aldosteronism. Clin Chem 2016; 62: 514-524

[77] Williams TA, Peitzsch M, Dietz AS et al. Genotype-Specific Steroid Profiles Associated With Aldosterone-Producing Adenomas. Hypertension 2016; 67: 139-145

[78] Scholl UI, Abriola L, Zhang C et al. Macrolides selectively inhibit mutant KCNJ5 potassium channels that cause aldosterone-producing adenoma. J Clin Invest 2017; 127: 2739-2750

[79] Caroccia B, Prisco S, Seccia TM et al. Macrolides blunt aldosterone biosynthesis: A proof-of-concept study in kcnj5 mutated adenoma cells ex vivo. Hypertension 2017; 70: 1238-1242

[80] Maiolino G, Ceolotto G, Battistel M et al. Macrolides for KCNJ5-mutated aldosterone-producing adenoma (MAPA): Design of a study for personalized diagnosis of primary aldosteronism. Blood Press 2018; $1-6$ 
[81] Takeda R, Matsubara T, Miyamori I et al. Vascular complications in patients with aldosterone producing adenoma in Japan: Comparative study with essential hypertension. The Research Committee of Disorders of Adrenal Hormones in Japan. J Endocrinol Invest 1995; 18 : 370-373

[82] Catena C, Colussi G, Lapenna R et al. Long-term cardiac effects of adrenalectomy or mineralocorticoid antagonists in patients with primary aldosteronism. Hypertension 2007; 50: 911-918
[83] lacobellis G, Petramala L, Cotesta D et al. Adipokines and cardiometabolic profile in primary hyperaldosteronism. J Clin Endocrinol Metab 2010; 95: 2391-2398

[84] Fischer E, Adolf C, Pallauf A et al. Aldosterone excess impairs first phase insulin secretion in primary aldosteronism. J Clin Endocrinol Metab 2013; 98: 2513-2520

[85] Somloova Z, Widimsky J Jr., Rosa J et al. The prevalence of metabolic syndrome and its components in two main types of primary aldosteronism. J Hum Hypertens 2010; 24: 625-630 